\title{
Téoros
}

Revue de recherche en tourisme

\section{Tourisme et musées : complémentarités interactives!}

\section{André Hut}

Volume 11, numéro 2, juillet 1992

Quand les musées s’ouvrent au tourisme...

URI : https://id.erudit.org/iderudit/1078093ar

DOI : https://doi.org/10.7202/1078093ar

Aller au sommaire du numéro

Éditeur(s)

Université du Québec à Montréal

ISSN

0712-8657 (imprimé)

1923-2705 (numérique)

Découvrir la revue

Citer cet article

Hut, A. (1992). Tourisme et musées : complémentarités interactives! Téoros, 11(2), 42-44. https://doi.org/10.7202/1078093ar d'utilisation que vous pouvez consulter en ligne.

https://apropos.erudit.org/fr/usagers/politique-dutilisation/ 


\title{
E U R - I N F O Tourisme et musées: complémentarités interactives!
}

\author{
André Hut ${ }^{*}$
}

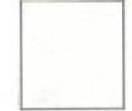

Le tourisme apporte aux musées une promotion supplémentaire bienvenue, davantage de visiteurs et donc plus d'entrées financières.

Les musées, quant à eux, vont à la rencontre des besoins de détente et de développement culturel des vacanciers, permettant ainsi au tourisme d'atteindre ses finalités socio-culturelles. D'où trois parties: les visiteurs, le tourisme, les musées.

\section{Visiteurs}

Les touristes de la troisième génération qui passent encore leurs congés payés sur les littoraux d'Europe ne se contentent plus seulement de la plage, de la mer et du soleil. Ils souhaitent y exercer des loisirs actifs et culturellement valorisants. De plus, ces vacanciers, à majorité d' origine citadine, partagent, de plus en plus, les nouvelles valeurs et sensibilités que véhicule la revendication d'une meilleure qualité de vie. Cela engendre de nouvelles pratiques culturelles et touristiques $^{(1)}$ : notamment, le raccourcissement des longs séjours des grandes vacances d"été au profit de deuxième ou même troisième vacances, d'où le développement du tourisme de proximité. À partir de leurs centres d'intérêts les plus variés et d"une curiosité davantage éveillée par les informations télévisuelles, les citoyens s'adonnent d'une manière croissante aux excursions, erronément dénommées atourisme d'un jour $\psi^{(2)}$. Professionnels et experts du tourisme, y compris le Touring-club de Belgique et les Communautés Européennes analysent ces nouveaux comportements, leurs incidences culturelles et conséquences $6 \mathrm{co-}$ nomiques.

Lefaitque les gens semettentpassionnément à la recherche de leurs origines et $s^{4}$ intéressent à retrouver leurs racines socio-culturelles, rurales ou ouvrières, interpelle a la fois les musées et le tourisme qui peuvent, partiellement, $y$ apporter une réponse.

\section{Tourisme}

Depuis 1972 déjà, le Commissariat général au Tourisme, animé par Arthur Haulot, issu du milieu ouvrier et du tourisme associatif, avait lancé des campagnes de promotion centrées, chaque année, autour d'un autre thème illustrant les patrimoines naturels ou culturels du pays: année des châteaux, des moulins, des abbayes, des parcs et jardins ou du folklore. L'unedeces années thématiques, dans le cadre du $400^{\circ}$ anniversaire de la naissance du peintre Rubens, offritl'occasion, une deuxième fois, en 1977, de mettre en valeur les musées des beaux-arts où dominent peintures et sculptures comme l'année 1959, une première fois déjà, l'avait brillamment réussi!

Récemment, l'OPT (Office de Promotion du Tourisme), établissement d'utilité publique pour la partie francophone de Belgique ${ }^{(3)}$, après avoir polarisécelle-ci sur \&La Belgique gourmande* (1990), ou sLa Belgique en fêtte» (1991), consacrait l'année 1992 aux attractions. En effet, l'association d'intérêt, *Attractions et tourismes, fondée en 1982, se préoccupe, entre autre, à faire connaitre les activités de ses 165 membres, notamment par un guide, annuel et gratuit, présentant aussi bien les centres récréatifs et parcs de loisirs que les grottes et réserves naturelles ou les châteaux et les trains touristiques, sans oublier les musćes évidemment, avec index récapitulatif par type d'attraction. La collaboration de la partie flamande du pays et l'appui du Touring Club ont permis d'éditer, cette année, un guide national, regroupant 300 attractions, tiré à plus d'un million d'exemplaires.

Le ministère du Tourisme de Hollande orchestrait a son tour, sa promotion en organisant, en 1988, une "Année des muscées", illustrée par un guide gratuit, et orientée en priorité vers ses compatriotes mais aussi en faveur de ses voisins belge et allemand tout proches,

D'ailleurs, les dernières campagnes annuelles, «Les Pays-Bas, plaisirs de l'eau" (1990), ou «Pays des fleurs $*$ recommandaient les musées contribuant à approfondir et comprendre ces facettes del' identité hollandaise: par exemple, le musée de la navigation à Amsterdam ou le musće Frans Hals de Haarlem où une exposition invitait les visiteurs à suivre sune route des bulbes» en admirant
Expert en tourisme, protesseur et animateur, Europe. 
des illustrations, tableaux et objetsd'art, sans omettre de faire connaiture leurs architectures et décorations de jardins historiques, véritables musées de plein-air, de réputation intemationale.

D' un tout autre style, l' Autriche, a la veille de fêter le millénaire de sa fondation en 1996 , prépare cet évênement par 4 années de wdécouverte de l'histoire et des réalisations des Habsbourgss dont l'empire s'étendait sur la moitié de l'Europe et dont maints membres de la famille ont régné à la tête de nombreuses nations durant six siecles! Un guide, de grande qualité, s'efforce de relever, à travers tout le pays, les sites les plus importants commémorant laprésenceet les oeuvres de la famille rếgnante. wSur les routes des Habsbourgs à travers 1'Autrichem ne reprend pas seulement une liste commentée des lieux mais offreaussi une interpréfation thématique regroupée respectivement autour des styles baroque, gothique ou renaissance du patrimoine monumental comme aussi des grandes découvertes techniques, industrielles ou botaniques dont le pays leur est redevable, le tout explicité par de nombreux musées existant à ces propos.

La Belgique diffuse, en outre, une brochure sur Les villes d'art, anciennes, nombreuses et renommées, proches les unes des autres, à 35 minutes en voiture ou en train à partir de Bruxelles; en plus d' informations pratiques sur les hebergements, voies d'acces et de communications, principaux événements, la brochure propose à la visite les musées aussi nombreux que variés.

L'Autriche, de son côte, distribue un guide sur les Arts en Autriche, richement illustré, après une intéressante présentation historique des périodes artistiques dans leur environnement culturel, des sigles permettent de retrouver les oeuvres d'art auxquelles el lesappartiennent dans la liste de présentation de celles-ci répertorićes géographiquement, région par région.

Enfin, de plus en plus, les organismes touristiques offrent des forfaits, de fin de semaine oudecourtes vacances, polarisés autour d'un centre d'intérêt. Bruxelles, par exemple, invite à goûter la bière, la bande dessinée ou l'art nouveau, grâce à des approches systémiques: promenades, visites d'entreprises, dégustations, renoontres avec des professionnels, etc., agrémentent et complètent la visite du musée respectif, pivot thématique.
Dans le même sens, des comités départementaux français de tourisme, appellent les visiteurs du Salonannuel des vacances à aller à la rencontre d'une région et de ses habitants, a la decouverte de son identité particulière, grâce à de courts séjours, soiten milieu rural à partir de parcs naturels et d'écomusées (champagne, cidre, camembert), soit d'archéologie industrielle ruinière, métallurgique ou textile.

Des collaborations touristiques s'amorcent d'ailleurs entre les pays voisins - France, Hollande, Allemagne, Grand-Duché du Luxembourg - de la Belgique vu que des liensgéographiques (fleuve, région naturelle, etc.) ou d'histoire commune les rapprochent. II faudra revenir sur ces expériences transfrontalières.

D'autre part, des Offices du Tourisme de grandes villes culturelles comme Paris ou Vienne ne se limitent plus, comme dans le passe, a orienter seulement les visiteurs vers les grands musées d'art, d'histoire ou des sciences de réputation mondiale mais proposent, à la carte, dans un guide vendu ou un feuillet gratuit, tous les musées de l'agglomération tant la faim se développe chez les touristes de retrouver les facettes les plus diverses de la vie quotidienne, des professions et des activités de leurs prédécesseurs.

Leséditeurs publiant descollections de guides touristiques suivent le pas. En plus des guides classiques par ville, région ou pays, ils offrent des guides thematiques. Le Guide des musées de France (1984), chez Bordas, par exemple, est remarquable non seulement parce qu'il propose à l'appétit culturel 1700 musées mais surtout parce qu' il répond aux centres d'interèts des visiteur's potentiels par quatre index des plus utiles: oì peut-on voir lescollections, ventilées par thèmes, où peuton retrouver les oeuvres de cents peinires et sculpteurs, artistes et hommes célèbres cités ou encore le répertoire des villes à musées. Dans un même souci d'aider les visiteurs à organiser leur itinéraire selon leurs souhaits, les Éditions de La Manufacture (Paris) se renouvellent en sortant le Guide du patrimoine rural, recensant 400 musées, $6 c 0-$ musées et collections d'agriculture (1991), ainsi que le Guide du parrimoine industriel, regroupant 1000 musées, planétarium, centres de cultures scientifiques et techniques (1990).

De même, en Belgique, les guides IPPA, des éditions Lannoq, comprennent un Guide des musées complétant ceux qui traitent de la nature, des abbayes ou des châteaux.

\section{Musées}

Selon l' ICOM, lemuś́e est aunétablissement permanent, sans but lucratif, au service de la communauté, de son développement culturel et accessible à touss ${ }^{(4)}$.

Or, trop souvent jusqu'à présent, les musées des beaux-arts, d'histoire ou des sciences naturelles, sont restés peu acoessibles culturellement à la population en général dont les enfants et les jeunes en particulier. La présentation des objets et les commentaires sont par trop tributaires d'une culture clitiste et universitaire, ce qui bloque lacommunication avec un public peu initié partageant une démarche différente de culture populaire. Cela explique les aréticences à pénétrer dans des lieux considéréscommesactís ou réservés à une poignése d'intellectuels ${ }^{(5)}$. Ils ont une connotation ennuyeuse et décourageante vu les souvenirs négatifs d'excursions scolaires obligatoires de sinistre memoire!

Heureusement que progressivement, des responsables de musées ont développé des activités pour faciliter l'approche du passé, qu'il soit domestique, artistique, archéologique ou industriel. C'est pourquoi les personnes sensibles tout autant à cette mission pédagogique que de l'attente des gens à retrouver leurs racines et à comprendre se sont regroupées dans la Commission Éducation et Actions culturelles dans les musćes, au sein de $\mathrm{I}^{*}$ Association francophone des musées de Belgique. Dans le tract déjà cité, Les musées? C'est gai?, et distribué à leur stand au Salon des vacanoes, elle invite les touristes àentrer par curiosité pour découvrir les objets façonnes par des gens et qui racontent leur histoire, comme aussi pour se laisser étonner par l'habilité de l'artisan.

La démarche se veut ludique, plaisante et sous forme de jeux, ce qui correspond d'ailleurs aux espérances de personnes en loisirs et en vacances. D'où, depuis 1989 , une opération Musée en famille, en collaboration avec l'importante Ligue des familleset diffusée par leur périodique Le ligueur, diffusé à plusieurs centaines de milliers d'exemplaires.

Elle se déroule le premier week-end de juin dans une trentaine de musées, afin de mettre les gens en appétit, tant qu'ils sont au pays, avant qu'ils ne partent en vacances, et qu'ils puissent d'ailleurs pratiquer durant celle-ci, 
l'opération se prolongeant tout l'été. Les animateurs promettent que la surprise, l'admiration et le plaisir seront au rendez-vous. Toute la famille doit pouvoir réaliser une visite en s'amusant grâce à des rallyes, jeuxenigmes ou d'observation, circuits-promenades ou de découvertes, contes racontés, etc.

Autre investissement culturel pour l'avenir: ales musées prennent un coup de jeunew, se produit, en 1992, pour la quatrième année consécutive à Bruxelles eten Wallonie. Elle veut donner la possibilité aux adolescents d'abandonner l'idée reçue que le musée est un lieu rigide et de s' intếresser au patrimoine culturel et aux arts plastiques. Ces deux journées des 18 et 19 mars ont connu un succès de foulepuisqueplus de 19000 jeunes yont participéet que 1800 élèves-animateurs ont créé les 145 animations différentes présentées dans les musées ${ }^{(6)}$. Il fallait imaginer puis réaliser des animations destinées à accrocher les jeunes. Des exemples? "Aux Musées Royaux d'Art et d'Histoire à Bruxelles, un délilé de mode égyptien antique; au Musée de Louvain-la-Neuve, le décodage par ordinateur de messages transmis parun tambour africain; au Musée des BeauxArts de Tourmai, la mise en scène d'un tableau. Par des disciplines passerelles comme aussi la vidéo, la cuisine, le dessin, etc., il s' agissait de montrer à ces jeunes que loin d'êtremort ou de faire mourir d'ennui, le patrimoine culturel est vivant et amusant, tout dépendant de l'oeil avec lequel on le regardes,

Dans le même esprit, en France, dans le cadre du bicentenaire, le ministère de la Culture a mis sur pied un vaste programme de sensibilisation en lançant l'Année de l'archéologie: de septembre 1989 à septembre 1990, plus de cent manifestations régionales à travers le pays et l'appel à visiter 600 musées archéologiques ainsique des terrains de fouille avec en plus la possibilité de rencontrer les scientifiques et animateurs responsables de celles-ci. Faut-il rappeler l'expérience originale française du remarquable Archéodrome, situé sur une aire de repos de l'autoroute du Sud, à Beaune.

Les services éducatifs des musćes sont très actifs également. Celui des Musées Royaux d'Art et d'Histoire vient de communiquer à la presse son programme pour la saison touristique: L'étéaumusée, regards "92. Une pochette regroupe 6 séries d'activités différentes dont notamment: 9 evisites commen- tées du dimanche dans les diverses extensions du musée disséminées dans le paysage bruxellois", ou 11 "visites-promenades qui invitent à poser un regard nouveau sur diverses collections d'histoire de l'antiquité, respectivement de l'Égypte à la Grèce ou à l'Islam»; des visites approprices pour les enfants et adolescents sur l'exploration du musée; mais aussi, depuis plusieurs années déjà, des stages de plusieurs jours, sous le titre de Dynamusée : grâce aux animatrices et aux méthodes actives, les $6 / 10$ ou $10 / 15$ ans pourront $s$ 'initier aux couleurs, formes, atmosphères, mentalités qui ont imprégné des siècles d'histoires fabuleuses et qui feront remonter le temps, cette année, l'un des thèmes étant Le voyage d'Ulysse, presqu' une bande dessinée. Plus original encore, le programme Invitation au voyage, propose 4 pays (Suède, Grèce, Chine et Vietnam) aux personnes soit qui envisagent d'entreprendre un voyage et de le préparer, soit, au retour, de l'approfondir pour mieux comprendre, soit tout simplementd'enrêverà travers un voyage imaginaire.

Même démarche ludique exercée parcemême musée, en 1991, dans le cadre d'une opération de promotion touristique basée sur les musées bruxellois où l'OPT proposait un forfait passeport pour Bruxelles de 5 visites $(-30 \%)$ : les animateurs invitaient soit à découvrir, en un circuit de deux heures, 30 des plus beaux chefs-d'oeuvre de leur collection, intitulés Les must, soit, en juilletet en août, le même menu appelé Le temps retrouvé où la vie quotidienne et la pensée philosophique et religieuse sont évoquées, en alternance, à partir des objets et leur contexte historique.

Finalement, comment expliquer l'énorme succès des grandes expositions temporaires sur les Celtes ou les Vikings (Paris 1992), comme aussi, en Hollande, sur Van Gogh en 1990 avec 1,25 million de visiteurs et un solde positif de 3 millions de dollars canadiens, ou Rembrandt avec 440000 entrées? En fait, la majorité des visiteurs ne fréquentent pas les musées mais se précipitent à ces expositions. N'est-ce pas à cause du thème: un peuple ou une personne? que cela appartient à leur passé? que ces artistes sont des Européens qui ont vécu dans plusieurs pays? que les oeuvres et objets, habituellement dispersés dans divers musées d'Europe sont, cette fois regroupés en un seul lieu et permettent ainsi une meilleure appréhension globale et synergique?
En conclusion, cette panoplie d'activités de tourisme culturel, durant et hors-saison surtout, outre le bénéfice culturel, sauvegarde et développe l'emploi et engendre des retombées économiques directes et indirectes non négligeables. $f$

\section{REFERENCES}

(1) André Hut, Mutations culturelles et pratiques touristiques, Téros, wol. 4, no 2, 1985, Montréal p. 21.

(2) AIEST, Les excursions de la journbe et leur impact, 38e congrès, vol. 29, aoút 1988 a Brtéda. St-Gall, Suisse,

(3) Andre Hut, Organisation politique et administra. tive, p. 8, et Chacun apporte sa pierre, p.17 in Téoros, supplément Automné 1987. QuébecCommunauté française, Montréal.

(4) Dossier Les Musées, Nouvelles du patrimoine, mai 1986, no B, Bruxelles, p. 3.

(5) Les musebos? C'est gai?, Musées Royaux d'Ant et d'Histoire, 1990, pare du cinquantenaire, 1040, Bruxelles.

(6) Jean Rebuffat, Dix neuf mille coups de jeune, Le Soir. Bruxelles, 9 avril 1992 